\title{
COMMUNICATION ET DYNAMIQUES D'HYBRIDATION DANS LES ORGANISATIONS DE TYPE LABORATOIRE VIVANT : UN REGARD CONSTITUTIF SUR LE DÉMARRAGE D'UN LIVING LAB AU SERVICE DES AÎNÉS
}

\author{
Dany Baillargeon ${ }^{1}$ et Hélène BruneaU ${ }^{2}$
}

Ancrée dans le paradigme de l'innovation ouverte, la méthodologie laboratoire vivant (living lab) met en relation chercheurs, représentants des secteurs public et privé et citoyens afin de créer des solutions innovantes à des problématiques complexes. À travers un partage mutuel de connaissances et d'expériences autour d'un but commun, ce mode d'organisation hybride suppose également de maintenir les intérêts de chaque acteur, ce qui vient avec son lot de tensions. À partir d'une analyse des figures (Cooren, 2013) animées dans des textes et des conversations entourant le démarrage d'un laboratoire vivant axé sur les enjeux du vieillissement, nous exposons différentes tensions émanant du processus d'hybridation, mais également le favorisant. Quatre enjeux sont particulièrement porteurs des tensions : le statut du laboratoire, son fonctionnement, la représentation des parties prenantes et la terminologie en usage. D'un point de vue constitutif, nous démontrons que les tensions liées à l'appartenance participent du processus d'hybridation, ce dernier étant fait de dynamiques de détachements/rattachements.

1 Dany Baillargeon est professeur au Département des Lettres et Communication à l'Université de Sherbrooke.

2 Hélène Bruneau poursuit la maîtrise en Communication à l'Université de Sherbrooke..

Recherches en communication, n47 - Article publié le 19/12/2018 
Depuis la fin des années 1990, une vague d'organisations s'est constituée autour de la philosophie de l'innovation ouverte, qui décentre le locus d'innovation, passant d'un épicentre strictement en interne vers une mise en relation de partenaires externes, multisectoriels et multidisciplinaires (Chesbrough et al., 2008). Le cas des laboratoires vivants (living labs) nous semble particulièrement intéressant. En sciences sociales, les laboratoires vivants (LV) se caractérisent par la mise en relation de chercheurs, de représentants des secteurs public et privé et de citoyens autour d'un but commun de façon à proposer des solutions innovantes à des problématiques complexes (ex. changement climatique, pauvreté) (Picard, 2011). Compte tenu de la nécessaire interaction pour aligner les intérêts et besoins des parties prenantes (von Geibler et al., 2017), la communication représente un enjeu d'importance. Or, outre les travaux s'intéressant aux outils et plateformes de communication et à la nécessité de diffuser besoins et solutions aux parties prenantes (Pfeffermann et al., 2013), très peu semblent concevoir que ces formes organisationnelles sont évoquées et maintenues dans et par les pratiques de communication (Schoeneborn et al., 2014, p. 286).

Notre proposition met en lumière, à travers l'implantation du Laboratoire vivant au service des aînés (LVASA) ${ }^{3}$ les tensions émergeant autour de quatre enjeux : statut, fonctionnement, participation et représentation des parties prenantes, terminologie. Dans ces enjeux, le choc entre des figures contradictoires, surtout liées au sentiment d'appartenance, crée des tensions qui peuvent « détacher» les acteurs de l'organisation (Cooren et al., 2013). Or ces tensions, en même temps qu'elles engendrent ces détachements, conduisent à créer de nouveaux attachements " au nom de » la philosophie du LVASA. Ainsi, d'un point de vue constitutif, le processus d'hybridation crée de nouvelles appartenances au fil des dynamiques de détachements/rattachements.

3 Le nom a été changé pour préserver l'anonymat. 


\section{Laboratoires vivants : définition, fonctionnement et tensions}

Les démarches en laboratoire vivant ont vu le jour vers la fin des années 1990 au MIT à Boston pour aborder des problèmes technologiques complexes dans les contextes réels d'utilisation. Au tournant des années 2000, les sciences sociales ont importé la philosophie pour l'appliquer, par exemple, en santé et autonomie (Picard, 2011), en réhabilitation et réadaptation, en urbanisme social (Franz, 2015), à la qualité de vie d'une communauté (Edwards-Schachter et al., 2012).

Bien qu'il existe différentes définitions des laboratoires vivants, cinq éléments-clés circonscrivent l'approche laboratoire vivant (Dubé et al., 2014 ; Eskelinen et al., 2015 ; Picard, 2011) : 1) un processus de cocréation impliquant 2) une collaboration intersectorielle, 3) avec des citoyens-usagers engagés au cœur de la démarche, 4) dans un système ouvert où le développement de solutions innovantes à des problématiques complexes se fait, 5) dans le contexte de vie réelle. Le processus de cocréation intersectorielle invite les parties prenantes à être à la fois apprenant et enseignant (Bradwell \& Marr, 2008), et ce, à toutes les étapes du processus (Bergvall-Kareborn \& Stahlbrost, 2009).

Cette cocréation force les LV à composer avec des tensions : déséquilibre dans le partage des bénéfices ou des extrants, orientations des parties prenantes inconciliables, déséquilibre dans la balance du pouvoir ou structure organisationnelle incompatible avec la philosophie des LV (Ansell \& Gash, 2007). Qui plus est, le cycle relativement court du processus d'innovation en laboratoire vivant - en moyenne deux ans - confronte les parties prenantes aux enjeux caractéristiques des phases de démarrage « $[\ldots]$ ce qui comprend l'établissement de relations, le partage de connaissances, l'exploration de l'ignorance et l'élaboration de nouveaux concepts » (Soile \& Virpi, 2016, p. 17. Notre traduction). 


\section{Aborder les tensions et l'hybridation par les CCO}

C'est à la rencontre des enjeux propres au LV et des tensions inhérentes à son mode d'organisation que nous posons la question suivante : comment l'implantation et l'appropriation d'une méthodologie LV génèrent-elles des tensions constitutives orientant l'hybridation intersectorielle/interdisciplinaire?

Pour y répondre, nous proposons de regarder ces tensions en nous appuyant sur la typologie de Smith \& Lewis (2011). Partant du principe que toute organisation doit composer avec des demandes multiples et divergentes, les auteurs proposent quatre catégories de tension associées aux activités fondamentales de l'organisation :

- l'apprentissage : tensions entre construire sur le connu et faire tabula rasa pour chercher la disruption ou l'innovation radicale ;

- l'appartenance : tensions entre la construction et le maintien identitaire individuel et l'identité de l'organisation ;

- l'accomplissement : tensions entre les différentes acceptions du succès (ce qui est considéré comme une réussite);

- l'organizing : tensions dans les différents processus nécessaires à la production.

Les tensions peuvent également survenir à la rencontre de ces quatre catégories fondamentales. Il en résulte ainsi une typologie comprenant dix tensions, schématisée en Annexe 1 (Cf. Smith \& Lewis 2011).

De sorte à aborder l'hybridation comme processus et non comme état, nous utilisons la proposition de Smith \& Lewis en conjonction avec les approches de la communication comme constitutive des organisations (CCO), plus particulièrement la ventriloquie (Cooren, 2012, 2013). En arguant que « toute organisation ne peut se maintenir, évoluer et fonctionner qu'en s'incarnant dans les actions et les interactions impliquant ses représentants, que ceux-ci soient des documents officiels, des employés, des dirigeants, des sites Web ou des porte-parole " (Cooren \& Robichaud, 2011, p. 141), les CCO permettent de concevoir les 
pratiques, les textes et les discours organisationnels comme des agents qui « font une différence ", à savoir qu'ils agissent sur le cours d'une situation et donnent une direction à l'organizing (Cooren \& Fairhurst, 2009) : par exemple, la solidarité comme valeur cardinale d'une organisation sera inscrite dans les textes et discours organisationnels (charte, guide de l'employé) ; celle-ci sera convoquée pour prendre une décision budgétaire qui favorisera, disons, les services à la population à l'augmentation du salaire des dirigeants. La ventriloquie (Cooren, 2012, 2013) met en avant une double animation des êtres en soutenant que les êtres humains animent constamment des figures - idées, principes, valeurs, idéologies, faits - dans leurs conversations : ils font dire ou font faire des choses à ces figures. Cette «présentification» des figures agit dans les interactions entre personnes, mais les figures peuvent être déléguées dans des textes et des conversations qui, à leur tour, deviennent les ventriloques de ces mêmes figures. Plus encore, les individus ne " manipulent » pas seulement les figures, tel un ventriloque ; ces figures agissent également sur le ventriloque, en ce sens qu'un individu attaché à une figure, par exemple « la solidarité », tend à agir conformément à celle-ci, et ce, de façon itérative et récurrente. L'attachement de figures incarnées et déléguées dans les textes et les discours forme des réseaux d'événements communicationnels qui constituent l'organisation (Blaschke et al., 2012).

Prendre le pas d'une approche ventriloque des tensions suppose d'observer des attachements contradictoires à des figures dans une situation donnée, créant une impression de dissonance (Cooren et al., 2013, p. 265). Cette dissonance peut paralyser voire entraver l'organizing. Or il est plus riche de concevoir cette tension comme créant, engendrant, produisant d'autres dynamiques organisationnelles, dont celle de transformer l'organisation (Putnam et al., 2016) ou, dans le cas qui nous occupe, de constituer l'hybridation. 


\section{Méthodologie}

Le Laboratoire vivant au service des aînés (LVASA) s'est donné pour mission de développer des solutions innovantes et pérennes face aux défis du vieillissement (habitation, mobilité, participation citoyenne, etc.). Il a été lancé à l'initiative d'une équipe interdisciplinaire de sept chercheurs d'un centre de recherche - sis dans un Centre intégré universitaire de santé et de services sociaux (CIUSSS) 4 - qui souhaitait « contribuer à la levée des cloisons entre les secteurs public, privé et citoyen (ex. décideurs, promoteurs, organismes communautaires) $»^{5}$. À la suite de l'obtention d'une subvention en juillet 2016, le laboratoire est officiellement constitué en août 2016 autour d'un comité de pilotage composé des sept chercheurs à la base du projet et d'un comité dit " élargi », composé de seize chercheurs, trois représentants d'associations d'aînés, trois représentants du milieu municipal et d'un représentant du secteur privé. Ce comité élargi oriente le travail du laboratoire, permet des maillages, définit les projets pertinents. Notre terrain, qui procède d'observations participantes - le premier auteur étant membre du comité de pilotage - et non participantes - la seconde auteure agissant comme observatrice -, couvre les grandes étapes de la mise en place du LVASA à partir de la demande de subvention (Tableau 1).

4 Au Québec, les CIUSSS regroupent les services de santé et les services sociaux de même territoire administratif.

5 Extrait de la demande de subvention qui a permis de structurer les activités du LVASA. 
Tableau 1. Chronologie des événements du LVASA (de juin 2016 à mars 2017)

\begin{tabular}{|c|c|c|}
\hline & Instance & Contenu \\
\hline 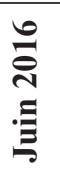 & $\begin{array}{l}\text { Comité de } \\
\text { pilotage }\end{array}$ & $\begin{array}{l}\text { Rédaction de la demande de subvention «Soutien à l'im- } \\
\text { plantation d'un LivingLab/Age Friendly-Community: } \\
\text { Pour transformer notre communauté amie des Aînés en } \\
\text { laboratoire vivant » }\end{array}$ \\
\hline$\underset{\substack{0 \\
\infty}}{\substack{\infty \\
\infty}}$ & $\begin{array}{l}\text { Comité } \\
\text { élargi }\end{array}$ & $\begin{array}{l}\text { Présentation du LV et de la démarche envisagée aux } \\
\text { membres présents. }\end{array}$ \\
\hline 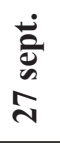 & $\begin{array}{l}\text { Comité } \\
\text { de pilotage }\end{array}$ & $\begin{array}{l}\text { Établissement d'une première proposition sur la mission- } \\
\text { vision-valeur (MVV) du LVADA à partir d'un document- } \\
\text { cadre de l'OMS. }\end{array}$ \\
\hline 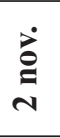 & $\begin{array}{l}\text { Comité } \\
\text { élargi }\end{array}$ & $\begin{array}{l}\text { Retour sur les éléments mission, vision et valeurs à la } \\
\text { suite des premières propositions basées sur l'OMS du } \\
\text { comité de pilotage. }\end{array}$ \\
\hline$\underset{\infty}{\stackrel{\dot{a}}{\Xi}}$ & $\begin{array}{l}\text { Comité de } \\
\text { pilotage }\end{array}$ & $\begin{array}{l}\text { Préparation de la présentation d'une présentation pu- } \\
\text { blique sur le LVADA. }\end{array}$ \\
\hline 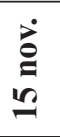 & $\begin{array}{l}\text { Présenta- } \\
\text { tion pu- } \\
\text { blique }\end{array}$ & $\begin{array}{l}\text { Présentation publique du projet LVADA pour intéresser } \\
\text { d'autres chercheurs à l'initiative. }\end{array}$ \\
\hline $\begin{array}{l}\stackrel{2}{\Xi} \\
\stackrel{2}{a}\end{array}$ & $\begin{array}{l}\text { Comité de } \\
\text { pilotage }\end{array}$ & $\begin{array}{l}\text { Préparation d'un appel de proposition de projets. Présen- } \\
\text { tation des acteurs en place et à venir. Enjeux de gouver- } \\
\text { nance avec une clarification des rôles des deux comités } \\
\text { (pilotage et élargi). }\end{array}$ \\
\hline 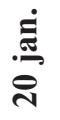 & $\begin{array}{l}\text { Comité de } \\
\text { pilotage }\end{array}$ & $\begin{array}{l}\text { Bilan sur les éléments du projet structurant vus en } 2016 . \\
\text { Retour sur les éléments MVV. }\end{array}$ \\
\hline 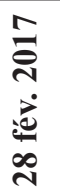 & $\begin{array}{l}\text { Comité } \\
\text { élargi }\end{array}$ & $\begin{array}{l}\text { Présentation des projets intra LV retenus et financés. } \\
\text { Discussion collective sur le nom et les éléments de MVV. } \\
\text { Recommandation sur la gouvernance du projet. }\end{array}$ \\
\hline
\end{tabular}

Afin d'aborder les tensions associées au processus d'hybridation du LVASA, nous avons analysé trois réunions de travail du comité de pilotage et deux réunions du comité élargi, pour un 
total de $14 \mathrm{~h}$ de captations audio. Les captations ont été transcrites de façon à pouvoir analyser plus aisément les matériaux. Notre méthode d'analyse a procédé en trois temps :

1. dépouiller les transcriptions de façon à relever les figures qui « font une différence », c'est-à-dire comment ces figures influencent le cours d'une discussion ou d'un processus ;

2. repérer les attachements contradictoires à certaines figures de façon à les associer à la typologie de Smith \& Lewis ;

3. reconstruire le réseau d'événements communicationnels que ces tensions engendrent sur le long terme et la façon dont elles changent le cours de l'organizing du LVASA.

\section{Présentation des résultats}

\section{1. Établissement de la mission, de la vision et des valeurs}

Dès la première rencontre axée sur la présentation du projet (8 sept.), les membres du comité élargi ressentent des difficultés à dégager les contours et la mission du LVASA : sa place dans le centre de recherche, son statut par rapport à d'autres laboratoires voire d'autres organismes communautaires. Lors de cette rencontre, Lynne et Eugénie, deux chercheuses instigatrices du projet, doivent composer avec l'incertitude provoquée par la forme et la structure inhabituelle du LVASA ${ }^{6}$.

1 Lynne : Moi j'aimerais ça demain, arriver, dire : ça va être, type A, 2 avec financement $\mathrm{B}$, pis outcome $\mathrm{Y}$. Mais là, ce serait mon projet, ce 3 ne serait pas le vôtre, là. Ce qui fait que c'est pour ça qu'il faut y aller 4 tranquillement. Pis, je pense qu'un des premiers, pis là je vais faire le $5 \quad$ lien avec le point qui s'en vient, un des premiers projets, éléments, qu'il 6 faut faire, c'est faire l'exercice qu'on est appelé à faire maintenant, à 7 savoir qu'est-ce qu'on veut avoir...c'est quoi la mission du...du... du...

8 de ce living lab, là, sans oublier qu'il y a aussi tout le volet financement

6 Le symbole « $=»$ et « [ $=»$ indique que deux tours de paroles se chevauchent. Par exemple, la phrase de Lynne à la ligne 13 chevauche celle d'Eugénie à la ligne 12, qui se poursuit à la ligne 14 . 
9 qui lui aussi est structurant. Tu sais, ce qui fait que... Oui,

10 Eugénie?

11 Eugénie [chercheuse] : Ben moi je pense que c'est ça, c'est que quand 12 tu parles d'allers et retours, moi je pense qu'effectivement, il faut peut-

13 être le faire comme ça. Je vois bien qu'en fait on n'est pas encore clair

14 sur la mission qu'on veut faire. Tu vois, quand on parle clinique, quand

15 on parle communautaire $=$

16 Lynne : [= C'est ça, c'est ça qu'il faut faire]

17 Eugénie : [ = même quand on parle communautaire, c'est dans quelle 18 mesure on est pas en train de refaire un organisme communautaire. Oui,

19 mais comment on se démarque du communautaire ? Est-ce que c'est

20 pas... bon, déjà, je pense que effectivement c'est pas clair. Si on com-

21 mence à réfléchir ça, j'irai un petit peu, peut-être dans ta voie, Lynne,

22 en disant: prenons des acteurs qui ne vont pas nous lier à la patte. Donc

23 c'est que, si par exemple, Denise [directrice du centre de recherche],

24 vient, puis tu en parles en clinique et puis après, ben on va être tellement

25 coincés avec la clinique qu'on va être coincés avec le CIUSSS et puis

26 avec tout ce qui le suit, et puis après. 'est peut-être trop tôt effectivement

27 tant qu'on n'a pas réfléchi à est-ce qu'on veut aller vers des services

28 cliniques potentiels...

29 Lynne : Peut-être qu'il y en aura, sûrement qu'il y en aura, mais ça va

30 peut-être passer davantage par les départements universitaires que par le

31 CIUSSS. De toute façon, ce que je pense qui est important c'est que ça

32 c'est la structure de gouvernance et là... moi, ce que je voudrais qu'on

33 ajoute rapidement des gens autres que des chercheurs [=

34 Denise (directrice) : [=Oui, ça on est d'accord.

35 Lynne : [=pour être capable d'avancer.

36 Denise : Ça c’est vrai !

Dans cet échange, plusieurs figures apparaissent et semblent s'entrechoquer. D'une part, Lynne défend que la mise en place d'un LV est un travail itératif jamais stabilisé, et dont la forme (lignes 1-10), le financement et les extrants ne sont jamais connus à l'avance, ce qui est contraire aux laboratoires cliniques habituels. Eugénie renchérit (lignes 11-15) en invoquant qu'il faut procéder par allers-retours pour clarifier la mission. Puis, elle mobilise la figure de la recherche clinique (lignes 19-23), pour y opposer la recherche du LVASA qui doit évoluer en marge de la recherche clinique au risque d'" être coincés avec le CIUSSS et puis avec 
tout ce qui le suit ». Lynne vient ajouter du poids (lignes 29-33) en soulignant que le LVASA est composé « d'autres gens, qui ne sont pas des chercheurs ", qu'il n'est pas un laboratoire de chercheurs. Dans cet échange, Lynne et Eugénie rappellent qu'elles sont attachées à la figure d'une recherche ouverte, libre, tout en rappelant le caractère interdisciplinaire du LVASA. Or la place traditionnelle de la recherche n'est pas difficile à laisser partir, certains revendiquent une structure organisationnelle qui puisse sécuriser autant que valider leur rôle, jusque dans la définition de la mission, comme le démontrent les résultats du sondage effectué auprès des membres concernant la mission (Figure 1).

\section{J'ajouterais à la mission :}

3 réponses

J'utiliserais le living lab comme un incubateur d'idées au service des chercheurs.

je ne vois pas la recherche ou le processus de recherche

Recherche évaluative participative

Figure 1. Commentaires sur l'énoncé de la mission du LVASA

\subsection{Mission et valeurs du LVASA}

De façon à clarifier la mission, le comité de pilotage se réunit à plusieurs reprises (27 sept., 2 nov., 20 janv., 28 février) afin de produire un énoncé de mission, vision et valeur (MVV) du LVASA à soumettre au comité élargi. Lors de la rencontre du 27 septembre, Lynne présente l'énoncé de MVV de l'Organisation mondiale de la santé (OMS) arguant que ce sont les orientations de l'OMS qui balisent les axes d'intervention des politiques sur le vieillissement à travers le monde, et donc l'orientation des fonds de recherche. Une fois l'ébauche terminée, le comité sollicite une rétroaction du comité élargi, avec un sondage, puis une discussion à la réunion du 2 novembre.

Ce travail de consultation a relevé des figures en contradiction. Entre autres, alors que l'OMS propose une orientation pour « vieillir en restant actif », cette notion se retrouve dans le premier 
descriptif de la mission du LVASA sous les termes de « vieillissement actif », ce qui avive du ressentiment dans le groupe :

Paul (chercheur) : Moi, mon commentaire c'est pourquoi faudrait mettre un qualificatif à vieillissement? Pourquoi on met quelque chose d'engagé à vieillissement? Parce que c'est vrai, après on tombe tout le temps un peu dans les nuances de qui parle, selon les politiques sociales, selon $[\ldots]$

Lynne: $\quad$ Chaque terme associé à vieillissement est aussi une posture sur le vieillissement.

Ce même malaise sur l'institutionnalisation ou l'idéologie de certains termes transparaît également dans le questionnaire, lorsque les membres du comité élargi ont été interrogés par voie du sondage en ligne concernant la mission du LVASA (Figure 2).

\section{J'apporterais les changements suivants à l'énoncé :}

4 réponses

À mon avis, l'objectif n'est pas assez clair, on veut « inspirer, développer et animer des mvts avec les aînés pour faire quoi au juste : afin d'améliorer la qualité de vie ? (comme le Angelab à Boston ?), augmenter le transfert recherche>société ?

le terme « vécu émancipant » me semble un peu vague et une mission doit être claire pour tous.

Bien que le français est parfait, je trouve l'énoncé fort complexe à comprendre.

La notion de « vécu » est très contestée en sciences sociales

Où est la recherche?

Figure 2. Commentaire sur l'énoncé de mission proposé aux membres du LVASA

Ces événements communicationnels déplient des figures de l'aîné, en tension entre des définitions institutionnelles (OMS), ou une « posture » clinique, et une vision plus humaniste en invoquant un « vécu émancipant ». Or ce « vécu » suscite l'opposition : 
plusieurs évoquent un terme « galvaudé » et « très pop psycho » dont l'usage ne clarifie pas la mission et questionne une vision de l'aîné (Figure 3), qui ne doit pas s'éloigner trop des « données probantes » de la recherche clinique.

\section{J'apporterais les changements suivants à l'énoncé : \\ 2 réponses}

à réfléchir : semble trop optimiste et non inclusif des personnes qui vont perdre de l'autonomie ou avec problèmes de santé ou sociaux ... peu importe la vision.

Je n'adhère pas à une vision optimiste si on n'a pas les données pour le promouvoir. Je suis plus dans une vision réaliste.

Figure 3. Commentaires sur l'énoncé de la vision du LVASA

Au final, après plusieurs discussions, la figure du vieillissement est débattue au cours de la réunion du comité de pilotage du 20 janvier. Le comité stabilise la mission et s'attache donc à une nouvelle « vision clairvoyante et engagée d'un vieillissement actif et en santé, basée sur la participation citoyenne des aînés et arrimée à leur situation de vie $»^{7}$. Cette figure de l'aîné se veut donc à la fois « réaliste/clairvoyante », rappelant la figure institutionnalisée de l'aîné " actif et en santé », mais qui se veut conséquente d'un vécu réel, « arrimé à leur situation de vie».

\subsection{Qui fait quoi : gouvernance et fonctionnement}

Nous l'avons entrevu, dans les échanges sur le statut du LVASA, le caractère intersectoriel et interdisciplinaire suscite quelques craintes et inconforts au regard de la gouvernance, particulièrement à l'égard de la place du privé :

1 Denise : Moi je suis allée... ((à Lynne)) t'as sûrement vu Silver Valley

2 en France, quand t'es allée la dernière fois ? C'est sûr que moi, jusqu'à

3 date, j'ai hâte de voir le modèle que nous ça va prendre. Parce que mon

4 inquiétude de ce que moi j'ai vu jusqu'à date, il y a une grosse emprise

7 Énoncé MVV présenté sur le site internet du laboratoire. 
5 du secteur privé sur, pour eux c'est la manne d'or ((validation groupe))

6 Les compagnies viennent voir les chercheurs, ils ont besoin d'un cher-

7 cheur pour leur développer des contenus, puis « développez-nous

8 quelque chose, nous autres on va aller faire de l'argent avec ça », puis

9 je n'ai pas vu beaucoup, beaucoup de places où le secteur public, l'en-

10 seignement, la recherche $=$

11 Lynne : [=Mais, mais, oui, les municipalités

12 Denise : [ = prenaient une place. Je trouvais que les autres ils prenaient

13 beaucoup, beaucoup de place...

14 Eugénie : Dans Silver Valley, ils ne se disent pas Living Lab, c'est un 15 consortium plus de...

16 Denise : Mouais, mais c'est un peu ça qu'ils font là.

17 Eugénie : Ben quand même, parce qu'en fait, moi, tu sais ce que je 18 reviens beaucoup à mon avis, c'est quand tu as mis tes trois places 19 [recherche, public, citoyen] et puis que tu dis il y a enseignement de part 20 et d'autre. Et puis c'est absolument ça qu'il faut avoir, c'est absolument 21 ça, où l'expertise doit être là.

On voit ainsi que la figure de l'entreprise privée incarne un danger, celui de phagocyter les fruits de leur travail ou de leur expertise (lignes 4-10). Eugénie contrecarre avec la figure de l'expertise, qui se construit à travers l'enseignement provenant des « trois places » (lignes 17-20), et qui fait l'autorité d'un acteur dans un LV. Ainsi, deux figures du privé s'entrechoquent : le privé mercantile et le privé expert, quand son « expertise doit être là » (ligne 21). D'ailleurs, parmi les partenaires privés qui joindront l'équipe du LVASA, notons une firme d'ingénieurs civils ayant à son actif des projets en transport durable et adapté. Ainsi, ce « privé expert » a pu s'attacher au groupe, puisque déjà empreint d'une volonté sociétale. 


\section{Discussions : l'appartenance comme principale source de tension}

Lorsque l'on rapatrie ces tensions en suivant la typologie de Smith et Lewis (voir Annexe 1), il est intéressant de constater que la plupart des tensions sont associées à des enjeux d'appartenance (Tableau 2).

Tableau 2. Intégration des espaces de tension du LVASA dans la matrice de Smith \& Lewis

\begin{tabular}{|c|c|c|}
\hline $\begin{array}{l}\text { Apprentissagel } \\
\text { appartenance } \\
\text { Terminologie et lan- } \\
\text { gage en usage (1) }\end{array}$ & Apprentissage & $\begin{array}{l}\text { Apprentissage/ } \\
\text { organisation }\end{array}$ \\
\hline $\begin{array}{l}\text { Appartenance } \\
\text { Représentation des } \\
\text { parties prenantes (2) }\end{array}$ & $\begin{array}{r}\begin{array}{r}\text { Appartenancel } \\
\text { organisation }\end{array} \\
\text { Fonctionnement (4) } \\
\text { accomplissement } \\
\text { Terminologie et } \\
\text { langage en usage (5) }\end{array}$ & Organisation \\
\hline $\begin{array}{l}\text { Accomplissement/ } \\
\text { appartenance } \\
\text { Statut du LVASA (3) }\end{array}$ & Accomplissement & $\begin{array}{l}\text { Accomplissement/ } \\
\text { organisation }\end{array}$ \\
\hline
\end{tabular}

Ainsi, tant dans l'établissement des énoncés MVV que dans les réflexions sur la gouvernance, ce qui importe et convoie son lot de tensions a trait aux mots, parfois lourds de sens, utilisés pour nommer les actions du LVASA (dans le tableau : "Terminologie et langage en usage »); à la place que l'on attribue aux différentes 
parties prenantes (« Représentation des parties prenantes »), ou à d'autres structures ou institutions (« Statut du LVASA »), à la façon dont celui-ci produira les innovations attendues («Fonctionnement »). Nous trouvons donc, en gras, les tensions identifiées lors de la période de démarrage du LVASA, que nous avons associées à la typologie de Smith \& Lewis (en italique).

Il va de soi que la participation et la représentation des parties prenantes (2) renvoient directement à la coexistence difficile à arrimer entre les identités individuelles et l'identité du groupe, alors que les individus sont aux prises avec un besoin de « se distinguer tout en recherchant une forme d'homogénéité avec le reste du groupe » (Smith \& Lewis, 2011, p. 383. Notre traduction). Les chercheurs, plus attachés à la figure traditionnelle du chercheur comme locus d'innovation, voient cette position replacée au même niveau que celui des autres membres du laboratoire, et l'autorité de l'expertise scientifique est particulièrement mise à mal. Un expert n'est expert que parce qu'il convoque un savoir qui le précède, est animé par celui-ci et se fait le ventriloque d'une communauté experte (Cooren, 2013, p. 111).

Cette communauté experte s'incarne à son tour dans la figure de l'aîné cliniquement ou institutionnellement définie, et qui devrait également apparaître dans le fonctionnement (4) : le laboratoire doit être au « service de la recherche » ou « des chercheurs » (appartenance/organisation).

La philosophie LV convoie une terminologie (5) qu'il faut apprendre en même temps qu'elle témoigne d'une certaine appartenance (apprentissage/accomplissement). Entrent alors en tension la nécessité de développer le langage de l'organisation en devenir, en même temps de pouvoir s'entendre dans le moment présent (Smith \& Lewis, 2011, p. 384). De fait, la façon dont on réfère aux aînés et à leur vécu, leurs expériences, leurs états, appelle ainsi un lien à une communauté de parole à laquelle on appartient : le comité de pilotage qui se sert des documents de l'OMS ou les personnes employant cette terminologie ventriloquent par le fait même ces institutions, qui viennent baliser certaines pratiques et certaines façons de conduire les actions organisationnelles (Sandhu, 2017) du LVASA. La manière de définir les aînés ou l'expertise (1) 
(appartenance/apprentissage) peut créer des tensions entre le désir de changer les façons de faire tout en maintenant une certaine cohérence identitaire (Smith \& Lewis, 2011, p. 384).

Ces " anciennes " définitions attachent les membres du LVASA à une façon de faire de la recherche au sein du laboratoire (5) (appartenance/accomplissement), qui est garante de succès certains, alors qu'elles doivent céder la place à des approches permettant de bâtir les connaissances nécessaires pour l'avenir (Smith \& Lewis, 2011, p. 384).

\section{Conclusion : l'hybridation comme appartenance / détachement/ attachement}

Ainsi, partant d'une lecture selon les approches $\mathrm{CCO}$, nous proposons que le processus d'hybridation - à tout le moins pour une organisation en début de constitution - en est un d'appartenance, que nous associons à des dynamiques de détachement/ attachement. Pour qu'il y ait hybridation, il faut d'abord, se détacher de certaines figures, donc, se délester d'une certaine appartenance, d'un attachement à certaines figures qui « constituent ce que nous sommes et qui nous sommes, ce que nous voulons ou ce que nous devons faire, ce que nous croyons ou attendons, etc. » (Cooren et al., 2013, p. 264. Notre traduction). Ensuite, l'hybridation opère lorsqu'il y a attachement à de nouvelles figures. Pour que la philosophie du LV soit mise en action, elle doit appartenir à ses membres, ceux-ci doivent y être attachés, de sorte qu'elle soit distribuée dans l'organisation (Bencherki \& Cooren, 2011). Ainsi, le renoncement - temporaire ou permanent - aux figures en contradiction durant l'implantation se fait au profit de nouveaux attachements : à la philosophie participative, au désir d'impliquer les aînés dans la recherche de solution, à un mode de recherche plus inclusif ; ces nouveaux attachements créent, par conséquent, de nouvelles appartenances. En somme, l'hybridation met en scène des tensions créatrices. Comme le soutiennent Putnam et al. (2016, p. 84), observer la façon dont se résolvent ces tensions permet de déchiffrer si ces tensions tendent à changer ou à reproduire des idéologies, des structures, des systèmes, créant « une sorte de 
continuité dans la rupture (parce que la rupture est déjà dans les choses), ou de rupture dans la continuité (parce que les choses ne sont pas données) »(Hennion, 2013, § 42). Bien que la présente étude couvre davantage la période d'implantation - et donc de détachements - du LVASA, les dynamiques de rattachement ouvrent un potentiel créatif : la stabilisation de la mission - incarnant un nouveau vocabulaire auxquels sont maintenant attachées les parties prenantes - de même que l'arrivée de partenaire du secteur privé constituent déjà des marques de cette potentialité.

\section{Références}

Ansell, C. \& Gash, A. (2007). Collaborative Governance in Theory and Practice. Journal of Public Administration Research and Theory 18(4), 543-571.

Bencherki, N. et Cooren, F. (2011). Having to be: the possessive constitution of organization. Human Relations 64(12), 1579-1607.

Bergvall-Kareborn, B. \& Stahlbrost, A. (2009). Living Lab: an open and citizen-centric approach for innovation. International Journal of Innovation and Regional Development 1(4), 356-370.

Blaschke, S., Schoeneborn, D. \&Seidl, D. (2012). Organizations as Networks of Communication Episodes: Turning the Network Perspective Inside Out. Organization Studies 33(7), 879-906. doi:10.1177/0170840612443459

Bradwell, P. \& Marr, S. (2008). Making the most of collaboration: An international survey of public service co-design. London: Demos.

Chesbrough, H., Vanhaverbeke, W. \& West, J. (2008). Open Innovation: Researching a New Paradigm. [Kindle version]. New York: Oxford University Press.

Cooren, F. (2012). Communication Theory at the Center: Ventriloquism and the Communicative Constitution of Reality. Journal of Communication 62(1), 1-20.

Cooren, F. (2013). Manières de faire parler : Interaction et ventriloquie. Lormont : Éditions du Bord de l'Eau.

Cooren, F. \& Fairhurst, G. T. (2009). Dislocation and stabilization: How to Scale Up from Interactions to Organization. Dans L. L. Putnam \& A. M. Nicotera (Eds.). Building Theories of Organization: The Constitutive Role of Communication (pp. 117-152). New York: Taylor \& Francis.

Cooren, F., Matte, F., Benoit-Barné, C. \& Brummans, B. (2013). Communication as Ventriloquism: A Grounded-in-Action Approach to the Study of Organizational Tensions. Communication Monographs 80(3), 255-277. doi:10.1080/03637751.20 13.788255

Cooren, F. \& Robichaud, D. (2011). Les approches constitutives. Dans S. Grosjean \& L. Bonneville (Eds.). La communication organisationnelle. Approches, processus et enjeux. Montréal : Chenelière Éducation.

Dubé, P., Sarrailh, J., Billebaud, C., Grillet, C., Zingraff, V. \& Kostecki, I. (2014). Le livre Blanc des Living Labs. Umvelt Service Design, Montréal, Canada. 
Edwards-Schachter, M. E., Matti, C. E. \& Alcántara, E. (2012). Fostering quality of life through social innovation: A living lab methodology study case. Review of Policy Research 29(6), 672-692.

Eskelinen, J., Garcia Robles, A., Lindy, I., Marsh, J. \& Muente-Kunigami, A. (2015). Citizen-Driven Innovation: A Guidebook for City Mayors and Public Administrators. World Bank and European Network of Living Labs.

Franz, Y. (2015). Designing social living labs in urban research. info 17(4), 53-66.

Hennion, A. (2013). D'une sociologie de la médiation à une pragmatique des attachements. Retour sur un parcours sociologique au sein du CSI. SociologieS.

Pfeffermann, N., Minshall, T. \& Mortara, L. (2013). Strategy and Communication for Innovation. Berlin, Heidelberg : Springer Berlin Heidelberg. Disponible à l'adresse : https://journals.openedition.org/sociologies/4353

Picard, R. (2011). Pertinence et valeur du concept de « Laboratoire vivant» (Living Lab) en santé et autonomie. Rapport CGEIET, no 2010/46/CGIET/SG, conseil général de l'industrie, de l'énergie et des technologies.

Putnam, L. L., Fairhurst, G. T. \& Banghart, S. (2016). Contradictions, Dialectics, and Paradoxes in Organizations: A Constitutive Approach. The Academy of Management Annals 10(1), 65-171.

Sandhu, S. (2017). Organization as Communication and Institutional Theory: Opportunities for Communicative Convergence? Dans S. Blaschke \& D. Schoeneborn (Eds.). Organization as Communication: Perspectives in Dialogue (chapitre 5). New York: Routledge.

Schoeneborn, D., Blaschke, S., Cooren, F., McPhee, R. D., Seidl, D. \& Taylor, J. R. (2014). The Three Schools of CCO Thinking: Interactive Dialogue and Systematic Comparison. Management Communication Quarterly 28(2), 285-316. doi: $10.1177 / 0893318914527000$

Smith, W. K. \& Lewis, M. W. (2011). Toward a theory of paradox: a dynamic equilibrium model of organizing. Academy of Management Review 36(2), 381-403.

Soile, J. \& Virpi, L. (2016). Enhancing Early Innovation in an Urban Living Lab: Lessons from Espoo, Finland. Technology Innovation Management Review 6, 17-26. von Geibler, J., Baedeker, C., Liedtke, C., Rohn, H. \& Erdmann, L. (2017). Exploring the German Living Lab Research Infrastructure: Opportunities for Sustainable Products and Services. Living Labs, 131-154. 
Annexe 1 : Catégories des tensions types (adapté de Smith \& Lewis, 2011)

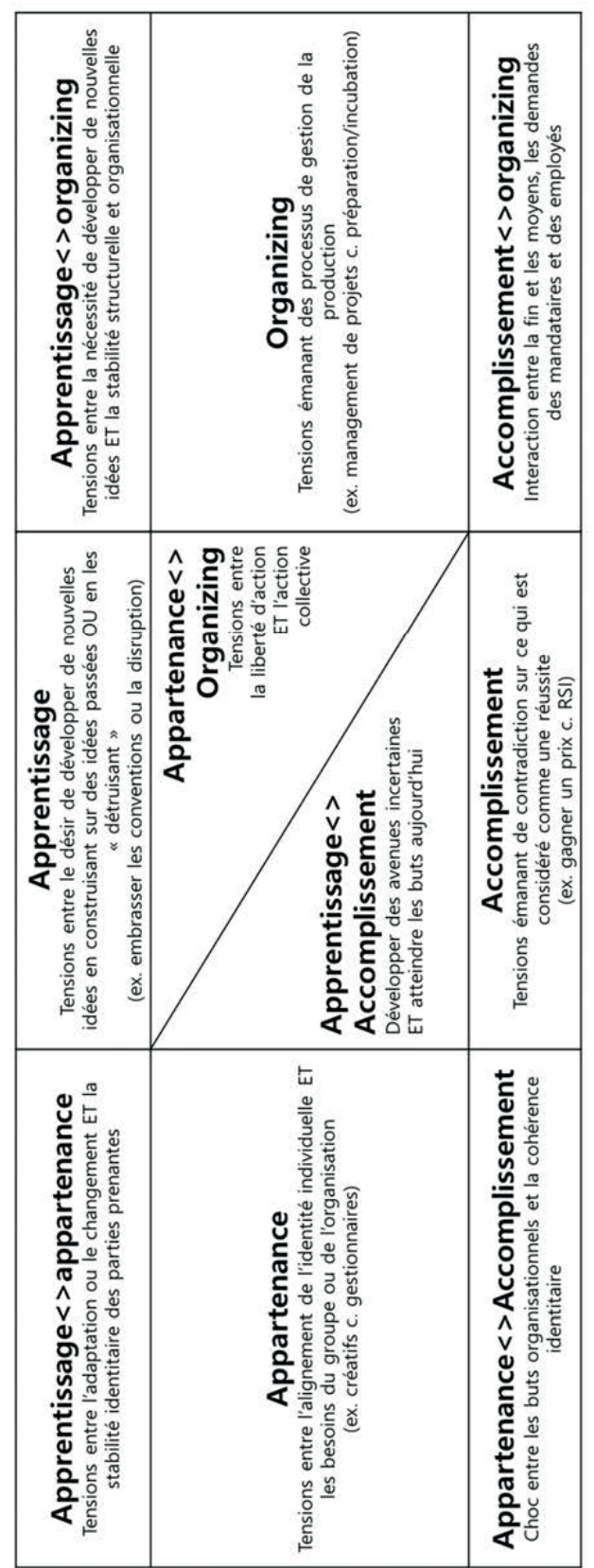




\section{(c) (1) $\Theta \Theta$ \\ (c) NC ND Publié sous la licence Creative Commons}

«Attribution - Pas d'Utilisation Commerciale - Pas de Modification 4.0 International» (CC BY-NC-ND) 